\title{
On Advances in Cancer Suicide-genes Therapy
}

\author{
A.R.Rama ${ }^{1,2 *}$, I. Zafra ${ }^{2}$, M. Burgos ${ }^{3}$ and J. Prados ${ }^{2}$ \\ ${ }^{1}$ Department of Health Science, University of Jaen, Jaen, Spain \\ ${ }^{2}$ Institute of Biopathology and Regenerative Medicine (IBIMER), University of Granada, Granada, Spain \\ ${ }^{3}$ Institute of Biotechnology and Department of Genetics, University of Granada, Granada, Spain
}

Received: 24 May, 2013; Accepted: 20 June, 2014, Published: 24 June, 2014

“Corresponding author: Ana Rosa Rama Ballesteros, Department of Health Science, University of Jaen, Jaen, Spain, Tel: +34699290855; E-mail: arama@ujaen.es

\begin{abstract}
Chemotherapy, radiotherapy and surgery are still the main cancer treatments. However, they carry considerable toxicity that often causes other adverse symptoms. The gene therapy intends to eliminate the tumor without damaging the normal host tissue. The classical suicide gene therapy needed the conversion of non-toxic prodrug into toxic metabolite and the bioavailability of active drugs. The discovery of genes that encode cytotoxic proteins overcomes these needs. There are many patents which describe the direct action of these new genes, and their uses as antitumor treatments. Some of the drawbacks of the tumor gene therapy are the low efficiency of in vivo gene delivery and the low tumor specificity. Advances on this field suggest the use of a combined gene therapy and chemotherapy or radiotherapy to increase the effectiveness of the antitumor treatment.
\end{abstract}

Keywords: Cancer; Suicide gene therapy; Combined therapy

\section{Introduction}

The aim of the conventional cancer therapy is to eliminate the tumor without damaging the normal host tissue. Chemotherapy, radiotherapy and surgery still remain as the main cancer treatments. However, they carry considerable toxicity that, often, causes other adverse symptoms. Nausea and emesis are some of the effects that the cisplatin-based chemotherapy may cause. An important problem after radiotherapy is the damage caused to the bone marrow. ${ }^{[1]}$ Gene therapy is presented as a possibility to modify or damage the tumor cell from within. In the last years, the gene therapy have been investigated in several fields, such as: immunomodulatory gene therapy, antiangiogenic gene therapy, RNA interference (rnai) therapy, genetic modulation of resistance or sensitivity, corrective and pro-apoptotic gene therapy and suicide gene therapy. ${ }^{[2]}$ In spite of those advances, the tumor gene therapy shows low efficiency of in vivo gene delivery and low tumor specificity. A combined gene therapy and chemotherapy ${ }^{[3,4]}$ and or radiotherapy ${ }^{[5,6]}$ has been proposed to increase the effectiveness of the antitumor treatment.

In this review, we show the current state of the cancer suicide gene therapy and its great potential in combination with chemotherapy and radiotherapy.

\section{Suicide gene therapy}

Suicide gene therapy is considered as a potential way to treat cancer. It is based on the use of genes from bacterial or viral origin, whose encoding enzymes are responsible for converting a nontoxic prodrug into toxic metabolites. ${ }^{[7,8]}$ The suicide gene therapy has been investigated in many cancer types: breast, glioblastoma, lung, hepatoma and bladder cancers. ${ }^{[9-12]}$

In this review, we will outline some of the suicide genes systems proposed for cancer therapy.

Cytosine deaminase: Cytosine deaminase (CD) catalyzes the deamination of the prodrug 5-fluorocytosine (5-FC), to lead to the toxic drugs 5-fluorouracil (5-FU). 5-FU inhibits the DNA and RNA synthesis, resulting in cell death. ${ }^{[13]}$ The CD/5-FC therapy has a potential clinical utility for lung ${ }^{[12]}$, gliomas ${ }^{[10]}$, neck squamous ${ }^{[14]}$, cervical ${ }^{[15]}$ and breast ${ }^{[11]}$ cancers. Mouse lung cancer models were used by Zarogoulidis et al. ${ }^{[10]}$, to study the antitumor efficacy of an adenovirus (Ad) vector-mediated CD followed by systemic 5-FC. Three groups, of 20 mice each, were studied: a control group without therapy, another group with intratumoral administration of 5-FC for 10 days; and another one with intratumoral administration of 5 -FC for 10 days plus Ad-CD on the day 2. The group treated with the Ad-CD plus 5-FC exhibited a greater decrease in the tumour volumes than either of the other two.

Also, the CD/-5-FC system enhances the effects of the radiotherapy. ${ }^{[16,17]}$ hela tumor xenografts were randomly divided into five groups: group A (control), group B (intratumoraly injected with and $\mathrm{Ad}-\mathrm{CD} / 5-\mathrm{FC}$ ), group $\mathrm{C}$ (radiation only) and group D (intratumoraly injected with and Ad-CD/5-FC and radiation). The groups $\mathrm{C}$ and $\mathrm{D}$ received a single (12.5 Gy) or fractionated (3 Gy x 5 days) radiation respectively. The group D showed a greater decrease in the tumor growth by up to 2.4fold $(\mathrm{P}<0.01)$ and 2.5 -fold $(\mathrm{P}<0.05)$, for 12.5 Gy or 3 Gy x 5 days respectively as compared to the group $\mathrm{C}^{[17]}$

Thymidine kinase: The Herpes Simplex Virus type 1 (HSV1) thymidine kinase (TK) converts non-toxic ganciclovir (GCV) into the toxic triphosphate ganciclovir (GCV-TP). GCV-TP is incorporated into DNA, stopping the replication and causing cell death. ${ }^{[13,18]}$ This mechanism of DNA damage is p53-dependent, 
and other genes such as ATM and p27 are also activated [19]. The HSV-TK/GCV system exhibited a promising approach for cancer suicide-gene therapy in lung ${ }^{[8]}$, breast ${ }^{[19]}$, hepatoma ${ }^{[20]}$ and bladder ${ }^{[21]}$ cancers. Current studies, with a Bifidobacterium infantis (BI) carrying HSV-TK plus GCV administration, showed inhibition of the bladder tumour in rat models. The rat models were randomly divided into three groups: injected with normal saline (group A), BI only (group B) and BI-TK (group C). The group $C$ evidenced the highest level of apoptosis, which was correlated with higher expression levels of Fas, fasl, Cyt-C and caspase-9. ${ }^{[21]}$

Several studies use both systems, HSV-TK/GCV and CD/5-FC, and have been shown to support a synergistic interaction. ${ }^{22-}$ ${ }^{24]}$ Huang et al. ${ }^{[25]}$, designed a double suicide gene system with CD/TK to study their bystander effects. Colon cancer cells were transfected with this double system and, after transfected, GCV or 5-FU prodrugs or both of them were administered. The results revealed a greater inhibition rate and a stronger bystander effect when both prodrugs were administered, in contrast with the use of a single prodrug.

Carboxypeptidase: Carboxypeptidase G2 (CPG2) activates carboxyl, phenol, or aniline mustard prodrugs, in which the DNA alkylating chain has been synthetically deactivated through $\mathrm{N}$-substitution of l-glutamate ${ }^{[25]}$, causing DNA-DNA interstrand cross-links and cell death. CPG2 has been used as antitumor therapy in breast, colon, hepatocellular and colon carcinomas. ${ }^{[26-}$ ${ }^{30]}$ The ZD2767P prodrug is a substrate of GPG2, which activates this prodrug as a bifunctional DNA interstrand cross-linking alkylating agent. This system has been used in hepatocellular carcinoma cells and xenografts exhibiting inhibition of tumour growth and extending the lifespan of the mice. ${ }^{[29]}$ Similar results have been shown in human colon carcinoma xenografts. ${ }^{[30]}$

According to this reaction, other prodrugs have been studied. [26-28] Oncolytic Salmonella typhimurium-derived bacterium VNP20009 was designed to deliver the CPG2. Human breast carcinoma, human colon carcinoma cells and B16-F10 mouse melanoma xenografts were injected with VNP2009/CPG2 and treated with several derivatives of L-glutamic prodrugs. CPG2 activated the prodrug and a significant decrease in the tumor growth of xenografts was observed. ${ }^{[28]}$ Two novel benzoic acid L-glutamate mustards, di- and trifluorinated prodrugs, are activated by CPG2, being cytotoxic for human breast carcinoma cells, while the tetra fluorinated prodrug is a competitive inhibitor of CPG2.[27]

Nitroreductase: The nitroreductase gene (NTR) from E. Coli, encodes an enzyme which is frequently used for transforming prodrugs into active drugs. NTR attaches a 4-nitrobenzyl group to a leaving group such as a phosphor amide or a carboxylate. 5-[aziridin-1-yl]-2,4-dinitrobenzamide (CB1954) is a prodrug of NTR, whose antitumor effect has been observed in human hepatocellular carcinoma and squamous carcinoma xenografs. ${ }^{\text {[31] }}$ A phase I/II clinical trial in 19 patients with prostate cancer analyzes the effectiveness of an adenovirus vector encoding NTR plus the systemic prodrug CB1954. Changes in the kinetic of the prostate-specific antigen (PSA) were used as indicators of tumor progression. $>10 \%$ PSA was decreased in five patients, and two patients showed $>50 \%$ decrease in 1 month, implying a reduction in the tumor size. ${ }^{[32]}$ Furthermore, the NTR-CB1954 system has an enhancer effect on radiotherapy. Ovarian cancer cells were stably transduced with the NTR gene. When they were treated with CB1954 and a single-fraction irradiation, the effect of radiotherapy was enhanced. These results were similar in human colon cancer xenograft. ${ }^{[33]}$

Other prodrugs for NTR have been tested as antitumor treatment for colon ${ }^{[34]}$, breast ${ }^{[35]}$ and lung ${ }^{[36]}$ carcinoma. 1-(2-methylpiperidin-1-yl) diazen-1-ium-1,2-diolate is a nitric oxide prodrug, which is converted to its active form in the presence of NTR. Sharma et al. ${ }^{[37]}$ added exogenously NTR to DLD-1 human adenocarcinoma cells, which were exposed to 1-(2-methylpiperidin-1-yl) diazen-1-ium-1,2-diolate. NTR transformed this prodrug into an active form and caused a high inhibition of the growth of the DLD-1 cells.

The previous systems have two fundamental limitations: the conversion of non-toxic prodrug into toxic metabolite and the bioavailability of active drug. ${ }^{[4,38,39]}$ These limitations are overcome by the use of genes that encode cytotoxic proteins, which have a direct antitumor action. The traditional therapy is targeted to cells undergoing division; however, this new therapy could be effective both in tumours with rapid development, and in tumours with slow growth. ${ }^{[3,4,40]}$

Gef: The gef gene encodes a membrane protein composed of 50 amino acids, which is anchored in the cytoplasmic membrane by its N-terminal..$^{[41]}$ In human tumour cells, gef induces apoptosis and cell cycle arrest, so it could be a complement to classical strategies such as chemotherapy. ${ }^{[3,38,42-44]}$ Prado's et al. [38], have transfected the gef gene into MCF-7, a human breast cancer cell line, to determine their combined effect with some cytotoxics. Four groups were established: a control group, a group transfected with $g e f$, a group treated with paclitaxel (Pac), docetaxel (Doc) or doxorubicin (Dox) at different concentrations, and a group treated with a combination of the gef gene and the cytotoxins. Reduction in cell growth was produced by all the cytotoxins, and the gef gene was able to induce a continuous decrease in the MCF-7 cell viability in the absence of any drug. Nevertheless, the combined therapy showed the largest growth inhibition supporting a strong synergistic effect. The combined therapy gef/Dox (10 um) had approximately 15\% more effect than the sum of both treatments. This effect may allow to reduce the concentration of drug in the treatment of breast cancer. Similar findings have been noted in colon and lung cancer. ${ }^{[3,43]}$ In both cancers, gef produced a significant decrease in cell growth, and its combination with some drugs of choice in the clinical treatment enhanced notably their effect. A $50 \%$ reduction of the cell growth was observed $24 \mathrm{~h}$ after transfection of the gef gene, and showed a 15-20\% enhancement of the antiproliferative effect when combined with the drugs. ${ }^{[3]}$ A study in lung multicellular tumour spheroids (MTS) revealed a decrease of MTS volume up to $87.4 \%$ when MTS was treated with $g e f / P a c$ versus untreated control MTS. ${ }^{[43]}$ 
$\boldsymbol{E}: E$ is a $\phi \times 174$ bacteriophage gene that encodes a 91-amino acid membrane protein with lytic function and a C-terminal domain that is involved in oligomerization. The E protein is a non-competitive inhibitor of the mray translocase of E.coli, which catalyzes the formation of lipid I, the first lipid intermediate in the synthesis of the cell wall ${ }^{[45]}$. Several studies demonstrated the antitumoral activity of the $E$ gene in lung, colon, melanoma and breast cancers. The expression of $E$ induced a decrease in cell growth producing an irregular distribution of cells in the monolayer cultures. Dilated mitochondrias with disrupted cristaes, a significant activation of caspase 3, and the release of cytochrome $\mathrm{C}$ into the cytoplasm $48 \mathrm{~h}$ after transfection, supported these results [Figure 1]. ${ }^{44,39,46]}$

The combined therapy with the $E$ gene and some cytotoxic drugs showed a greater inhibition of cell proliferation than the treatment with either the $E$ gene or the drugs alone, showing a synergistic effect as described for the gefgene. The growth of A-549 and T-84 cultured cells decreased a $19.8 \pm 1.3 \%$ and $19.2 \pm 1.7 \%$ respectively after $E$ gene transfection, and the combination with some cytotoxins increased the inhibition of proliferation. E/ Pac therapy induces up to $85 \%$ of growth inhibition in A-549 lung cells. This level of growth inhibition is only reached with the highest concentration of Pac alone. ${ }^{[39]}$ Studies with MCF-7 MTS corroborated this enhancement of proliferation inhibition. The volume of sferoids decreased an $18.9 \pm 2.3 \%$ after $E$ gene transfection. The E/Dox combination caused greater percentage of volume decrease $(63.9 \pm 2.7 \%)$ than Dox $(35.1 \pm 1.5 \%)$ or $E$ gene $(18.9 \pm 1.0 \%)$ treatments alone. Thus, in this case, it is also possible to reduce the dose of the drugs to achieve a safe and effective treatment, reducing side effects. ${ }^{[46]}$

Apoptin gene: The Chicken Anemia Virus VP3 protein, also known as Apoptin, has a nuclear localization in tumor cells but not in "normal" cells. This allows a specific induction of apoptosis in cancer cells. ${ }^{[47]}$ This ability to kill only tumor cells has been reported in breast ${ }^{[48]}$, head and neck ${ }^{[49]}$, prostate ${ }^{[50,51]}$, lung cancer, osteosarcoma ${ }^{[50]}$, hepatocellular ${ }^{[52]}$ and nasopharyngeal

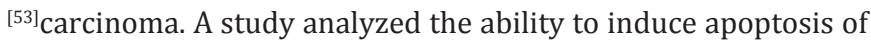
five truncated apoptin proteins in human breast adenocarcinoma MCF-7 cells and human liver Chang cells as control. Three out of the five truncated proteins targeted the nucleus of the MCF-7 cells initiating apoptosis and showing no effect on normal cells. ${ }^{[48]}$

Dacarbazine is an agent used in the treatment against malignant melanoma. When combined with apoptin they have demonstrated a synergistic antitumor effect in human and mouse B16-F1 melanoma cells. ${ }^{\left[{ }^{[4]}\right.}$ Similar findings were reported by Olijslagers et al. ${ }^{[0]}$, which indicated that the cytotoxicity of paclitaxel was enhanced by the combination with apoptin in several cancer cells. The combination of apoptin with photodynamic therapy or radiotherapy has a better response than the therapies alone. ${ }^{[49,53]}$

E1A: The early region $1 \mathrm{~A}(E 1 A)$ gene of the human adenovirus type 5 is a tumor suppressor and induces apoptosis in tumor cells. E1A has four conserved domains (CR1-CR4). CR1 domain is needed for its angiogenesis-inhibition, and CR2 domain is needed for the induced cell apoptosis and its chemosensitization activity. ${ }^{[55]}$ The antitumor activity of E1A has been tested in patients with breast, lung, prostate, bladder, ovarian and pancreatic cancers. ${ }^{[56]}$ E1A enhances the sensitivity of the cancer cells to some chemotherapeutic agents like paclitaxel, etoposide (VP16), cisplatin, 5-FU or gemcitabine. ${ }^{[57,58,59,60]}$ Bhattacharyya et al. [55], studied the combination of a mutated E1A CR2 domain (dl922-947), with a defective pRb binding, with gemcitabine or 5-FU agents, in human pancreatic cancer cell. The combination of d1922-947 with either agent demonstrated to kill more cells than when administered alone. The greatest synergistic effect was observed with the d1922-947/5-FU combination.

E1A has also been proved to enhance the radiosensitivity of human nasopharyngeal cancer in nude mice. Mice tumors treated with the combination E1A/radiotherapy were 4.7-fold smaller than those treated only with radiotherapy and 5.3-fold smaller than those treated only with E1A. ${ }^{[61]}$ Similar findings have been demonstrated in human cervical, hepatocellular carcinoma and glioblastoma. ${ }^{[62-64]}$

\section{Patents}

The current studies about cancer suicide-genes therapy clearly show its enhancing effect. Some advances in this field have been patented. An example is "Cancer gene therapy based

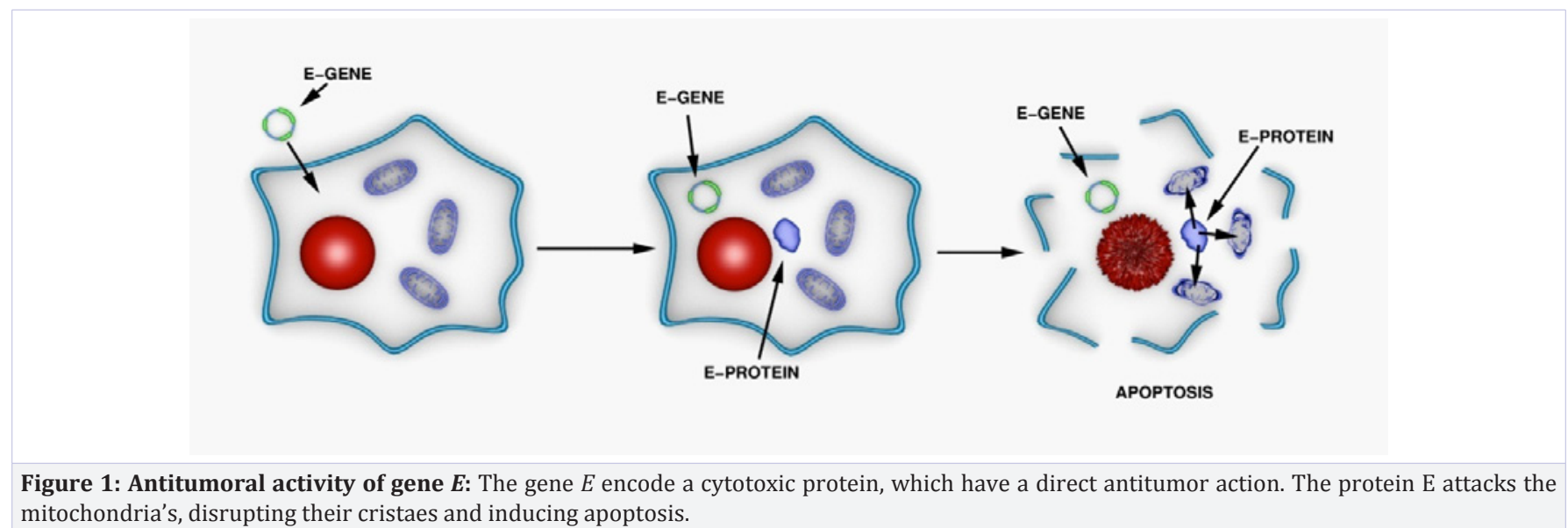

mitochondria's, disrupting their cristaes and inducing apoptosis. 
on translational control of a suicide gene" ${ }^{[65]}$ in which TK mRNA was joined to the $5^{\prime}$ UTR of the basic fibroblast growth factor. This gene is translationally regulated by eIF4E, which shows high levels in most solid tumors, causing the translation of TK mRNAs in cancer cells, but not in normal cells, suggesting an enhanced sensitivity to GCV.

Another patent is entitled "Polypeptide having an improved cytosine deaminase activity".[66] A native $\mathrm{CD}$ was modified by addition of an amino acid sequence. This new CD showed an improved CDase activity in LoVo human cells (adenocarcinoma of colon) transfected with the new-CD/5-FC system as compared to the original $\mathrm{CD} / 5-\mathrm{FC}$ system.

The use of the $E$ gene as a suicide gene for cancer gene therapy was registered in the patent "E gene for antitumor therapy". ${ }^{[67]}$ Tumors induced by B16-F10 (melanoma cells) in mice and treated with the $E$ gene exhibited a decrease of the initial tumour volume and a significant reduction as compared to the tumor control.

A research group has two patents about the use of the apoptin. "Apoptin-associating protein" describes the apoptotic activity of the apoptin when p53 is completely or partially non-functional. ${ }^{[68]}$ A year later, they registered the other one: "Modifications of apoptin". ${ }^{[69]}$ This suggests the use of the phosphorylation state of apoptin as a marker of cancer cells.

\section{Conclusion}

As shown in this review, suicide gene therapy has proved to be an effective treatment against cancer. The classic suicide gene therapy consists of the conversion of non-toxic prodrugs into toxic metabolites. The TK/GCV and the CD/5-FC systems are the most extensively studied, and other suicide genes, as $C P G$ or $N T R$, are on the way. The discovery of genes that do not need the use of a prodrug, but can kill directly has been a breakthrough in this therapy. Good examples are: the Gef protein, which induces apoptosis and cell cycle arrest; the E protein, which performs its antitumoral activity through mitochondrial apoptosis. Apoptin protein, that only induces apoptosis in cancer cells, and does not affect normal cells; and the E1A protein, whose CR1 domain inhibits angiogenesis, while its CR2 domain induces cell apoptosis and chemosensitization. Promoting research on these genes may provide a great advance on future cancer gene therapy.

Currently, the research on suicide gene therapy is focused on their combination with commonly used anticancer drugs, allowing the development of new drugs and suicide-genes-drugs combinations to decrease their toxicity and side effects and enhance their antitumoral activity.

\section{Acknowledgements}

Our work was funded by FEDER, Plan Nacional de Investigación Científica, Desarrollo e innovacióntecnológica (I+D+I), Instituto de Salud Carlos III (FIS) through projects no. PI11/01862 and PI11/02571; and by the Consejería de Salud de la Junta de Andalucía through project no. PI-0338.

\section{References}

1. Curigliano G, Cardinale D, Suter T et al. Cardiovascular toxicity induced by chemotherapy, targeted agents and radiotherapy: ESMO Clinical Practice Guidelines. Annals of oncology2012;23(7):vii155-vii166.

2. Cao S, Cripps A, Wei MQ. New strategies for cancer gene therapy: progress and opportunities. Clin Exp Pharmacol Physiol2010;37(1):108-114.

3. Ortiz R, Prados J, Melguizo C et al. Gef gene therapy enhances the therapeutic efficacy of cytotoxics in colon cancer cells. Biomed Pharmacother2012;66(7):563-567.

4. Rama AR, Prados J, Melguizo C et al. E phage gene transfection associated to chemotherapeutic agents increases apoptosis in lung and colon cancer cells. Bioeng Bugs2011;2(3):163-167.

5. Son CH, Keum JH, Yang K ert al. Synergistic enhancement of NK cellmediated cytotoxicity by combination of histone deacetylase inhibitor and ionizing radiation. Radiation Oncology2014;9:49.

6. Wenke L, Peng L, Xing W et al. Treatment of brain glioblastoma multiforme with pcdna3.1-Egr. 1p-p16 combined with gamma knife radiation: an experimental study on nude mice. Neurol India2013;61(5):491-496.

7. Duarte S, Carle G, Faneca H, de Lima MC, Pierrefite-Carle V. Suicide gene therapy in cancer: where do we stand now? Cancer Lett2012;324(2):160-170.

8. Leinonen HM, Ruotsalainen AK, Maatta AM et al. Oxidative stressregulated lentiviral TK/GCV gene therapy for lung cancer treatment. Cancer Res2012;72(23):6227-6235.

9. Kang NH, Hwang KA, Kim Su et al. Potential antitumor therapeutic strategies of human amniotic membrane and amniotic fluid-derived stem cells. Cancer Gene Ther2012;19(8):517-522.

10. Miller CR, Williams CR, Buchsbaum DJ, Gillespie GY. Intratumoral 5-fluorouracil produced by cytosine deaminase/5-fluorocytosine gene therapy is effective for experimental human glioblastomas. Cancer Res2002;62(3):773-780.

11.Yi BR, Hwang KA, Aboody KS, Jeung EB, Kim SU, Choi KC. Selective antitumor effect of neural stem cells expressing cytosine deaminase and interferon-beta against ductal breast cancer cells in cellular and xenograft models. Stem Cell Research2014;12(1):36-48.

12.Zarogoulidis P, Chatzaki E, Hohenforst-Schmidt W et al. Management of malignant pleural effusion by suicide gene therapy in advanced stage lung cancer: a case series and literature review. Cancer Gene Ther2012;19(9):593-600.

13. Kang NH, Hwang KA, Yi BR et al. Human amniotic fluid-derived stem cells expressing cytosine deaminase and thymidine kinase inhibits the growth of breast cancer cells in cellular and xenograft mouse models. Cancer Gene Ther2012;19(6):412-419.

14. Kwon SK, Kim SU, Song JJ, Cho CG, Park SW. Selective delivery of a therapeutic gene for treatment of head and neck squamous cell carcinoma using human neural stem cells. Clin Exp Otorhinolaryngol 2013;6(3):176-183.

15. Kim HS, Yi BR, Hwang KA, Kim SU, Choi KC. Anticancer effects of the engineered stem cells transduced with therapeutic genes via a selective tumor tropism caused by vascular endothelial growth factor toward hela cervical cancer cells. Mol Cells2013;36(4):347-354.

16. Kaliberov SA, Market JM, Gillespie GY et al. Mutation of Escherichia coli cytosine deaminase significantly enhances molecular chemotherapy of human glioma. Gene Ther2007;14(14):1111-1119. 
17. Liu J, Harada H, Ogura M, Shibata T, Hiraoka M. Adenovirus-mediated hypoxia-targeting cytosine deaminase gene therapy enhances radiotherapyin tumour xenografts.Brjcancer2007;96(12):1871-1878.

18. Predina JD, Judy B, Aliperti LA et al. Neoadjuvant in situ genemediated cytotoxic immunotherapy improves postoperative outcomes in novel syngeneic esophageal carcinoma models. Cancer Gene Ther2011;18(12):871-883.

19. Li H, Xiang S, Ma N, Hu W, Zeng Z (2011) [DNA damage caused by suicide gene therapy system under Tet-On regulation in breast cancer cells]. Zhong Nan Da Xиe Xиe Bao Yi Xue Ban2011;36(9):836-843.

20. Kim HA, Nam K, Lee M, Kim SW (2013) Hypoxia/hepatoma dual specific suicide gene expression plasmid delivery using bioreducible polymer for hepatocellular carcinoma therapy. J Control Release2013;171(1):1-10.

21. Yin X, Yu B, Tang Z et al. Bifidobacterium infantis-mediated HSV-TK/ GCV suicide gene therapy induces both extrinsic and intrinsic apoptosis in a rat model of bladder cancer. Cancer Gene Ther2013;20(2):77-81.

22. Hagen S, Baumann T, Wagner HJ et al. Modular adeno-associated virus (raav) vectors used for cellular virus-directed enzyme prodrug therapy. Scientific reports 2014;4:3759.

23. Huang Z], Feng QZ, You J et al. [Cytosine deaminase and thymidine kinase double suicide gene system driven by carcinoembryonic antigen promoter for the treatment of colorectal carcinoma xenograft in nude mice]. Zhonghua yi хие za zhi 2013;93(28):2235-2240.

24. Niu Y, Li JS, Luo XR. Enhancement of expression of survivin promoterdriven $\mathrm{CD} / \mathrm{TK}$ double suicide genes by the nuclear matrix attachment region in transgenic gastric cancer cells. Gene2014; 534(2):177-182.

25. Jamin Y, Eykyn TR, Poon E, Springer CJ, Robinson SP. Detection of the Prodrug-Activating Enzyme Carboxypeptidase G2 Activity with Chemical Exchange Saturation Transfer Magnetic Resonance. Mol imaging Biol 2013;16:152-157.

26. Capucha V, Mendes E, Francisco AP, Perry MJ. Development of triazene prodrugs for ADEPT strategy: new insights into drug delivery system based on carboxypeptidase G2 activation. Bioorg Med Chem Lett2012;22(22):6903-6908.

27. Davies LC, Friedlos F, Hedley D et al. Novel fluorinated prodrugs for activation by carboxypeptidase G2 showing good in vivo antitumor activity in gene-directed enzyme prodrug therapy. J Med Chem2005;48(16):5321-5328.

28. Friedlos F, Lehouritis P, Ogilvie L et al. Attenuated Salmonella targets prodrug activating enzyme carboxypeptidase $\mathrm{G} 2$ to mouse melanoma and human breast and colon carcinomas for effective suicide gene therapy. Clin Cancer Res2008;14(13):4259-4266.

29. Schepelmann S, Hallenbeck P, Ogilvie LM et al. Systemic genedirected enzyme prodrug therapy of hepatocellular carcinoma using a targeted adenovirus armed with carboxypeptidase G2. Cancer Res 2005;65(12):5003-5008.

30. Schepelmann S, Ogilvie LM, Hedley D et al. Suicide gene therapy of human colon carcinoma xenografts using an armed oncolytic adenovirus expressing carboxypeptidase G2. Cancer Res2007;67(19):4949-4955.

31. Djeha AH, Hulme A, Dexter MT et al. Expression of Escherichia coli B nitroreductase in established human tumor xenografts in mice results in potent antitumoral and bystander effects upon systemic administration of the prodrug CB1954. Cancer Gene Ther2000;7(5):721-731.
32. Patel P, Young JG, Mautner V et al. A phase I/II clinical trial in localized prostate cancer of an adenovirus expressing nitroreductase with CB1954 [correction of CB1984]. Mol Ther2009;17(7):1292-1299.

33. White CL, Menghistu T, Twigger KR et al. Escherichia coli nitroreductase plus CB1954 enhances the effect of radiotherapy in vitro and in vivo. Gene Ther2008;15(6):424-433.

34. Green LK, Syddall SP, Carlin KM et al. Pseudomonas aeruginosa nfsb and nitro-CBI-DEI--a promising enzyme/prodrug combination for gene directed enzyme prodrug therapy. Mol cancer2013;12:58.

35.Sekar TV, Foygel K, Willmann JK, Paulmurugan R. Dual-therapeutic reporter genes fusion for enhanced cancer gene therapy and imaging. Gene Ther2013;20(5):529-537.

36. Michaelsen SR, Christensen CL, Sehested M et al. Single agent- and combination treatment with two targeted suicide gene therapy systems is effective in chemoresistant small cell lung cancer cells. $J$ Gene Med2012;14(7):445-458.

37. Sharma K, Sengupta K, Chakrapani H. Nitroreductase-activated nitric oxide (NO) prodrugs. Bioorganic \& Medicinal Chemistry Letters2013;23(21):5964-5967.

38. Prados J, Melguizo C, Rama AR et al. Gef gene therapy enhances the therapeutic efficacy of doxorubicin to combat growth of MCF-7 breast cancer cells. Cancer Chemother Pharmacol2010;66(1):69-78.

39. Rama AR, Prados J, Melguizo C et al. E phage gene transfection enhances sensitivity of lung and colon cancer cells to chemotherapeutic agents. Int J Oncol2010;37(6):1503-1514.

40. Prados J, Alvarez PJ, Melguizo C et al. How is gene transfection able to improve current chemotherapy? The role of combined therapy in cancer treatment. Curr Med Chem2012;19(12):1870-1888.

41. Poulsen LK, Refn A, Molin S, Andersson P. Topographic analysis of the toxic Gef protein from Escherichia coli. Mol Microbiol1991;5(7):1627-1637.

42. Boulaiz H, Alvarez PJ, Prados J et al. Gef Gene Expression in MCF7 Breast Cancer Cells is Associated with a Better Prognosis and Induction of Apoptosis by p53-Mediated Signaling Pathway. Int J Mol Sci2011;12(11):7445-7458.

43. Prados J, Melguizo C, Rama A et al. Combined therapy using suicide gef gene and paclitaxel enhances growth inhibition of multicellular tumour spheroids of A-549 human lung cancer cells. Int $J$ Oncol2008;33(1):121-127.

44. Prados J, Melguizo C, Ortiz R et al. Regression of established subcutaneous B16-F10 murine melanoma tumors after gef gene therapy associated with the mitochondrial apoptotic pathway. Exp Dermatol2010;19(4):363-371.

45.ZhengY,StruckDK,YoungR.Purificationandfunctionalcharacterization of phix174 lysis protein E. Biochemistry2009;48(22):4999-5006.

46. Rama AR, Prados J, Melguizo C et al. Synergistic antitumoral effect of combination E gene therapy and Doxorubicin in MCF-7 breast cancer cells. Biomed Pharmacother2011;65(4):260-270.

47. Danen-Van Oorschot AA, Zhang YH, Leliveld SR et al. Importance of nuclear localization of apoptin for tumor-specific induction of apoptosis. J Biol Chem2003;278(30):27729-27736.

48. Shen Ni L, Allaudin ZN, Mohd Lila MA, Othman AM, Othman FB. Selective apoptosis induction in MCF-7 cell line by truncated minimal functional region of Apoptin. BMC cancer2013;13:488.

49. Schoop RA, Verdegaal EM, Baatenburg de Jong RJ, Noteborn MH. 
Apoptin enhances radiation-induced cell death in poorly responding head and neck squamous cell carcinoma cells. Basic Clin Pharmacol Toxicol2010;106(2):130-134.

50. Olijslagers SJ, Zhang YH, Backendorf C, Noteborn MH. Additive cytotoxic effect of apoptin and chemotherapeutic agents paclitaxel and etoposide on human tumour cells. Basic Clin Pharmacol Toxicol2007;100(2):127-131.

51. Zhang M, Wang J, Li C, et al. Potent growth-inhibitory effect of a dual cancer-specific oncolytic adenovirus expressing apoptin on prostate carcinoma. Int J Oncol2013;42(3):1052-1060.

52. Yuan L, Zhang L, Dong X et al. Apoptin selectively induces the apoptosis of tumor cells by suppressing the transcription of HSP70. Tumour Biol2013;34(1):577-585.

53. Fang X, Wu P, Li J et al. Combination of apoptin with photodynamic therapy induces nasopharyngeal carcinoma cell death in vitro and in vivo. Oncol Rep2012;28(6):2077-2082.

54. Jin JL, Gong J, Yin TJ et al. PTD4-apoptin protein and dacarbazine show a synergistic antitumor effect on B16-F1 melanoma in vitro and in vivo. Eur J Pharmacol2011;654(1):17-25.

55. Deng J, Kloosterbooer F, Xia W, Hung MC. The NH(2)-terminal and conserved region 2 domains of adenovirus E1A mediate two distinct mechanisms of tumor suppression. Cancer research2002;62(2):346-350.

56. Hemminki O, Diaconu I, Cerullo V et al. Ad3-htert-E1A, a fully serotype 3 oncolytic adenovirus, in patients with chemotherapy refractory cancer. Mol Ther2012;20:1821-1830.

57. Bhattacharyya M, Francis J, Eddouadi A, Lemoine NR, Hallden G. An oncolytic adenovirus defective in prb-binding (dl922947) can efficiently eliminate pancreatic cancer cells and tumors in vivo in combination with $5-\mathrm{FU}$ or gemcitabine. Cancer gene therapy2011;18(19):734-7433.

58. Chang CY, Lin YM, Lee WP, Hsu HH, Chen EI. Involvement of Bcl-X(L) deamidation in E1A-mediated cisplatin sensitization of ovarian cancer cells.Oncogene2006;25(18):2656-2665.
59. Ingemarsdotter CK, Baird SK, Connell CM, Oberg D, Hallden G, mcneish IA. Low-dose paclitaxel synergizes with oncolytic adenoviruses via mitotic slippage and apoptosis in ovarian cancer. Oncogene 2010;29(45):6051-6063.

60.Zhou RR, Jia SF, Zhou Z, Wang Y, Bucana CD, Kleinerman ES. Adenovirus-E1A gene therapy enhances the in vivo sensitivity of Ewing's sarcoma to VP-16. Cancer Gene Ther2002;9(5):407-413.

61.Zhou R, Chen J, Xiao Z. [Effect of adenovirus-E1A gene therapy on in vivo radiosensitivity to nasopharyngeal cancer]. Zhong Nan Da Xue Хиe Bao Yi Xиe Ban2009; 34(8):744-751.

62. Grunwald GK, Klutz K, Willhauck MJ et al. Sodium iodide symporter (NIS)-mediated radiovirotherapy of hepatocellular cancer using a conditionally replicating adenovirus. Gene Ther 2013;20(6):625-633.

63. Shen LF, Chen J, Zeng S et al. The superparamagnetic nanoparticles carrying the E1A gene enhance the radiosensitivity of human cervical carcinoma in nude mice. Mol Cancer Ther2010;9(7):2123-2130.

64. Valero ML, Cimas FJ, Arias L et al. E1a promotes c-Myc-dependent replicative stress: implications in glioblastoma radiosensitization. Cell Cycle2014;13(1):52-61.

65. Debenedetti A, Defatta RJ, inventor; Board of Supervisors of La. State Un. \& Agricultural and Mechanical College., assignee. Cancer gene therapy based on translational control of a suicide gene. United States patent US 6759394.2004.

66. Erbs P, inventor; Transgene SA assignee. Polypeptide having an improved cytosine deaminase activity. United States patent US 8226954.2012.

67. Prados JC, Melguizo C, Ortiz R, Rama AR, Boulaiz H, Aránega J. Gene E for antitumour treatment. Google.2009.

68. Danen-Van OAAAM, Noteborn MHM, Rohn JL, Toschi L, Weiss B inventor. Apoptin-associating protein. Google Patents.2001.

69. Noteborn MHM, Rohn JL, Mumberg D, Donner P, inventor. Modifications of apoptin. United States patent US 7319034.2008. 